ISSN 0103-9954

\title{
ESTRUTURA E POTENCIAL FUTURO DE UTILIZAÇÃO DA REGENERAÇÃO NATURAL DE FLORESTA DE VÁRZEA ALTA NO MUNICÍPIO DE AFUÁ, ESTADO DO PARÁ
}

\author{
STRUCTURE AND FUTURE POTENTIAL OF USE OF THE NATURAL REGENERATION IN \\ UPLAND FLOODPLAIN FOREST IN AFUÁ COUNTY, PARÁ STATE
}

\author{
João Ricardo Vasconcellos Gama ${ }^{1}$ Soraya Alvarenga Botelho ${ }^{2}$ \\ Michelliny de Matos Bentes-Gama ${ }^{3}$ José Roberto Soares Scolforo ${ }^{4}$
}

\begin{abstract}
RESUMO
O presente estudo teve como objetivo analisar a estrutura e indicar as probabilidade de utilização futura da regeneração natural de uma floresta não-explorada de várzea alta localizada no município de Afuá $\left(0^{\circ} 09^{\prime} 24^{\prime}\right.$ S e $50^{\circ} 23$ ' $12^{\prime}$ W), ao norte do estado do Pará. Foram amostradas 29 subparcelas de $100 \mathrm{~m}^{2}$ nas quais se mediu a altura $(\mathrm{h})$ de árvores e palmeiras $\operatorname{com} \mathrm{h} \geq 0,30 \mathrm{~m}$ até o diâmetro a 1,30 $\mathrm{m}$ do nível do solo (DAP) $<15 \mathrm{~cm}$, e o diâmetro das árvores compreendidas entre $\mathrm{h} \geq 3,0 \mathrm{~m}$ até DAP $<15 \mathrm{~cm}$. A densidade total foi 30.969 indivíduos/ha, pertencentes a setenta espécies, 57 gêneros e 25 famílias botânicas, com índice de diversidade de Shannon (H') de 2,68. As espécies mais importantes foram: Virola surinamensis, Euterpe oleracea, Astrocaryum murumuru, Geonoma laxiflora e Guarea guidonia. Muitas espécies são utilizadas como alimento pela fauna local, além de servirem como fonte de madeira e/ou, de produtos florestais não-madeireiros; algumas destas funcionam como complementação à dieta alimentar dos ribeirinhos, tais como: Eschweilera coriacea, Gustavia augusta, Inga Alba, Nectandra cf. risi e Protium spruceanum.
\end{abstract}

Palavras-chave: regeneração natural, fitossociologia, manejo florestal, Amazônia.

\section{ABSTRACT}

The aim of this paper was to analyze the structure and describe the future potential of use of the natural regeneration in an non-exploited upland floodplain forest located at EMAPA forestlands, Afuá County $\left(0^{\circ} 09^{\prime} 24\right.$ " S and $\left.50^{\circ} 23^{\prime} 12^{\prime \prime} \mathrm{W}\right)$, North of Pará State. The sample consisted of 29 sub-plots of $100 \mathrm{~m}^{2}$. In each sub-plot, all trees and palms with height $(\mathrm{h}) \geq 0.30 \mathrm{~m}$ and diameter at $1.30 \mathrm{~m}$ above ground level $(\mathrm{DBH})<15 \mathrm{~cm}$ were identified and measured. All trees with $\mathrm{h} \geq 3.0 \mathrm{~m}$ and $\mathrm{DBH}<15.0 \mathrm{~cm}$ were measured too. The total density was 30,969 individuals/ha distributed into 70 species, 57 genera and 25 botanical families, with a Shannon Index $\left(\mathrm{H}^{\prime}\right)$ of 2.68. The most important species were: Virola surinamensis, Euterpe oleracea, Astrocaryum murumuru, Geonoma laxiflora e Guarea guidonia. There are many species used for the local fauna as feeding, and many that also provide timber and non-timber forest products; some of them function as an addition to the diet of the riverine people, such as: Eschweilera coriacea, Gustavia augusta, Inga Alba, Nectandra cf. risi e Protium spruceanum.

Key words: natural regeneration, phytossociology, forest management, Amazonia.

\section{INTRODUÇÃO}

A Amazônia brasileira é a maior extensão de floresta tropical do mundo. A variedade dos recursos naturais ali existentes só ocorre em conseqüência das diferentes associações vegetais que crescem sob a influência de fatores ambientais intrínsecos a cada ecossistema que forma esse bioma. Entre os ecossistemas, que compõem a floresta equatorial amazônica, está a várzea cuja importância ecológica e socioeconômica para a região é marcante desde o processo de ocupação inicial até os dias de hoje, mantendo

1. Engenheiro Florestal, M.Sc., Doutorando do Departamento de Engenharia Florestal, Universidade Federal de Viçosa, CEP 36571-000, Viçosa (MG).jrvgama@vicosa.ufv.br

2. Engenheira Florestal, Dr., Professora do Departamento de Ciências Florestais, Universidade Federal de Lavras, Caixa Postal 37, CEP 37200-000, Lavras (MG). sbotelho@ufla.br

3. Engenheira Florestal, M.Sc., Pesquisadora da Embrapa Rondônia, BR 364, km 5,5, Caixa Postal 406, CEP 78900970, Porto Velho (RO). mbgama@cpafro.embrapa.br

4. Engenheiro Florestal, Dr., Professor do Departamento de Ciências Florestais, Universidade Federal de Lavras, Caixa Postal 37, CEP 37200-000, Lavras (MG). scolforo@ufla.br

Recebido para publicação em 5/09/2002 e aceito em 28/05/2003. 
populações ribeirinhas que praticam agricultura, pesca, e extrativismo de madeira e produtos florestais nãomadeireiros.

O ecossistema várzea localiza-se em áreas inundáveis da bacia amazônica cujos solos são constantemente renovados por causa da sedimentação natural que ocorre durante o período em que permanecem submersos. Nesse ambiente, desenvolvem-se plantas adaptadas fisiologicamente e morfologicamente que apresentam como característica marcante a madeira mais leve que as de espécies de terra firme (Prance, 1979).

Nas várzeas do estuário amazônico, a exploração da madeira e do palmito de açaí está entre as atividades econômicas mais importantes da região. Essas florestas começaram a ser exploradas economicamente no início da década de 50, época em que os primeiros trabalhos de inventário florestal já acenavam para a necessidade da inclusão da análise da estrutura da regeneração natural para um melhor conhecimento do estoque florestal. Ultimamente tem se destacado na legislação florestal brasileira (IBAMA, 2002) que a geração de informações sobre o estoque da regeneração natural é imprescindível à elaboração de planos de manejo florestal sob regime sustentado.

O termo regeneração natural, como estoque florestal, é conceituado por Finol (1971) como toda planta descendente de plantas arbóreas que se encontra entre $0,10 \mathrm{~m}$ de altura até o limite de $10 \mathrm{~cm}$ de diâmetro à altura do peito (DAP). Rollet (1969) considerou como regeneração natural todos os indivíduos com DAP $<5 \mathrm{~cm}$, entretanto, não limitou níveis de inclusão para seu estudo, ao dizer que indivíduos em classes de tamanho menores podem ser considerados como regeneração natural de indivíduos maiores.

A regeneração natural refere-se às fases iniciais de estabelecimento e desenvolvimento das plantas, sua boa condição quantitativa e qualitativa possibilita a preservação, a conservação e a formação de florestas, tanto de proteção integral como de uso sustentável. O entendimento dos processos de regeneração natural de florestas passa pelo conhecimento de informações básicas de caracterização da vegetação, além disso, a análise estrutural da regeneração natural é de suma importância para o planejamento do manejo e para a aplicação de práticas silviculturais direcionadas ao aproveitamento contínuo da floresta que vão favorecer o crescimento e maximizar o volume das espécies desejáveis por unidade de área.

Carvalho (1982) esclarece que a análise da estrutura da regeneração fornece a relação e a quantidade de espécies que constituem o estoque da floresta, suas dimensões e sua distribuição na comunidade vegetal, fornecendo dados que permitem previsões sobre o comportamento e o desenvolvimento da floresta no futuro. Oliveira (1995) confirma que essas informações são importantes ao silvicultor, a fim de conduzir a densidade das espécies comerciais e a qualidade da estrutura da floresta. Seitz (1988) enfatiza que, ao se estudar a regeneração natural, aumentam os custos do inventário, mas, por outro lado, se melhora o nível de informação sobre as associações, potencial produtivo e a dinâmica das espécies, tornando possível compensar o investimento.

O presente estudo teve como objetivo analisar a estrutura e descrever as possibilidades de utilização futura da regeneração natural de uma floresta não-explorada de várzea alta.

\section{MATERIAL E MÉTODO}

\section{Área de estudo}

O estudo foi realizado na propriedade florestal da Exportadora de Madeiras do Pará Ltda. - EMAPA, no município de Afuá ( $0^{\circ} 09^{\prime} 24^{\prime}$ ' S e 50 23' 12” W), estado do Pará. A área total é de 1.200 ha, dos quais 80 ha são de floresta de várzea baixa explorada e 1.120 ha de floresta de várzea alta. $\mathrm{Na}$ área de várzea alta, 500 ha foram utilizados em projetos de enriquecimento florestal e os demais 620 ha foram reservados a programas de manejo florestal. O solo é do tipo aluvional, os valores médios anuais de precipitação e temperatura correspondem, respectivamente, a $2.500 \mathrm{~mm}$ e $26^{\circ} \mathrm{C}$ (SUDAM, 1984).

\section{Amostragem e coleta dos dados}

No mapa da área, foram delimitados dez talhões de 60 ha $(600 \times 1.000 \mathrm{~m})$ e sorteou-se um deles para o inventário florestal, no qual foram distribuídas, sistematicamente, 29 parcelas de $20 \times 250 \mathrm{~m}(0,5 \mathrm{ha})$ no sentido Norte-Sul, onde cada uma destas continha uma subparcela de $10 \times 10 \mathrm{~m}$ para o inventário da

Ciência Florestal, v. 13, n. 2, 2003 
regeneração natural.

Nas subparcelas, foram mensurados todos os indivíduos das espécies arbóreas e palmeiras com altura $(\mathrm{h}) \geq 0,3 \mathrm{~m}$ e diâmetro a $1,30 \mathrm{~m}$ de altura do solo (DAP) $<15,0 \mathrm{~cm}$, dos quais foram anotadas as seguintes informações: nome regional; altura total; diâmetro dos indivíduos com $\mathrm{h} \geq 3,0 \mathrm{~m}$ até DAP $<15 \mathrm{~cm}$; forma de vida: árvore ou palmeira, arbusto, herbácea e cipó; e qualidade da regeneração natural: viva em pé, viva caída e com danos.

As espécies também foram classificadas segundo a sua categoria de uso em: comerciais comercializadas no mercado nacional e internacional; potenciais - comercializadas no mercado local e regional e não-comerciais. Todas as espécies amostradas tiveram seu material botânico coletado e passaram pelos processos de herborização. Posteriormente, foram identificadas quanto à família, gênero e espécie, por especialistas do Laboratório de Botânica da Embrapa Amazônia Oriental, em Belém, PA. Adotou-se o sistema de classificação de Cronquist (1988).

\section{Análise dos dados}

Todas as espécies foram classificadas de acordo com seu grupo ecológico, considerando-se, para tal, a proposta de Oliveira-Filho (1994), modificada para esse estudo com base em revisões bibliográficas e observações de campo. Foram adotadas as seguintes categorias: pioneira, clímax exigente de luz e clímax tolerante à sombra.

A suficiência amostral foi definida por meio do procedimento REGRELRP - Regressão Linear com Resposta em Platô, do Sistema para Análises Estatísticas - SAEG v. 5.0 da Universidade Federal de Viçosa (Nappo, 1999).

Para a análise da estrutura horizontal da regeneração natural, os parâmetros considerados foram: densidade, freqüência, dominância e índice de valor de importância os quais estão descritos em Curtis e McIntosh (1951).

No estudo da estrutura vertical da regeneração natural, foram utilizadas as classes de tamanho (CT) sugeridas pela FAO (1971), sendo: classe CT1: 0,3 m $\leq \mathrm{h}<1,5 \mathrm{~m}$; CT2: 1,5 m $\leq \mathrm{h}<3,0 \mathrm{~m}$; CT3: $\mathrm{h} \geq 3,0 \mathrm{~m}$ até DAP $<5,0 \mathrm{~cm}$; CT4: 5,0cm $\leq \mathrm{DAP}<10,0 \mathrm{~cm}$; e CT5: 10,0cm $\leq \mathrm{DAP}<15,0 \mathrm{~cm}$. As fórmulas empregadas para a estimativa das classes absoluta e relativa de tamanho da regeneração natural foram propostas por Finol (1971).

Para a definição das espécies mais importantes da fitocenose, todos os parâmetros citados foram reunidos na estimativa do índice de valor de importância ampliado para a regeneração natural (IVIARN), obtido pela expressão:

IVIARN $_{\mathrm{i}}=$ IVIRN $_{\mathrm{i}}+\mathrm{CRTRN}_{\mathrm{i}}$

Em que: IVIRN $_{\mathrm{i}}=$ Índice de valor de importância para a i-ésima espécie; e $\mathrm{CRTRN}_{\mathrm{i}}=$ Classe relativa de tamanho da regeneração natural para a i-ésima espécie.

A diversidade foi estimada pelo Índice de Shannon (H') calculado pelo logaritmo neperiano, e o padrão de distribuição espacial foi definido pelo Índice de Morisita (Ludwig e Reynolds, 1988), considerando-se, nesse caso, apenas as espécies que ocorreram em pelo menos duas parcelas.

\section{RESULTADOS E DISCUSSÃO}

\section{Composição florística}

Foram amostradas 70 espécies, 57 gêneros e 25 famílias botânicas (Tabela 1). Entre as famílias com maior riqueza de espécie, destacaram-se: Leguminosae (16), Arecaceae (7), Chrysobalanaceae e Clusiaceae (5). A ocorrência dessas famílias com o maior número de espécies foi compatível com o encontrado em floresta de várzea na Amazônia por Rodrigues (1961), Bentes e Maciel (1994) e Macedo (1996).

As cinco famílias que apresentaram maior porcentagem de indivíduos amostrados foram: Myristicaceae (33,5\%), Arecaceae (30,6\%), Leguminosae (6,7\%), Chrysobalanaceae (6,3\%) e Melastomataceae (3,5\%). Os gêneros que apresentaram o maior número de espécies foram: Licania (4), Inga

Ciência Florestal, v. 13, n. 2, 2003 
(3), Astrocaryum, Eugenia, Ormosia, Protium, Swartizia e Virola (2).

$\mathrm{Na}$ classificação por grupos ecológicos, verificou-se que 11,5\% das espécies eram pioneiras, 47,1\% clímax exigente de luz e 41,4\% clímax tolerante à sombra. Relacionando-se os grupos ecológicos com as categorias de uso das espécies, verificou-se que das oito espécies comerciais apenas uma era pioneira, e sete eram clímax exigentes de luz. Entre as 12 espécies potenciais, apenas uma era pioneira, seis clímax exigentes de luz, e cinco clímax tolerantes à sombra.

TABELA 1: Espécies amostradas em floresta de várzea alta na propriedade florestal da EMAPA, município de Afuá, Pará. Em que: Grupo de uso (GU) - comercial (C), potencial (P) e não comercial (NC); Grupo ecológico (GE) - pioneira (PI), clímax exigente de luz (CL) e clímax tolerante à sombra (CS); Padrão de distribuição espacial da espécie (PDE) - agregado (AG) e aleatório (AL); Classe de tamanho (CT) e Índice de valor de importância ampliado (IVIARN).

TABLE 1: Species sampled in the upland floodplain forest, at EMAPA forestlands, Afuá County, Pará State. Where: Category of use (GU) - commercial (C), potential (P), and non-commercial (NC); Ecological species group (GE) - pioneer (PI), light demanding (CL) and shade tolerant (CS); Spatial distribution pattern (PDE) - clustered (AG) and randomized (AL); Size class (CT); and Extended value of importance index (IVIARN).

\begin{tabular}{|c|c|c|c|c|c|c|c|c|c|c|}
\hline \multirow{2}{*}{$\begin{array}{l}\text { Família Botânica e } \\
\text { Nome Científico }\end{array}$} & \multirow{2}{*}{ GU } & \multirow{2}{*}{ GE } & \multirow{2}{*}{ PDE } & \multicolumn{6}{|c|}{ Número de Plantas/ha } & \multirow{2}{*}{ IVIARN } \\
\hline & & & & CT 1 & CT 2 & CT 3 & CT 4 & CT 5 & Total & \\
\hline ACANTHACEAE & & & & & & & & & & \\
\hline $\begin{array}{l}\text { Trichanthera gigantea Gleason } \\
\text { ANACARDIACEAE }\end{array}$ & $\mathrm{NC}$ & PI & $\mathrm{AG}$ & 86,2 & 17,2 & & 3,5 & & 106,9 & 2,46 \\
\hline Anacardium giganteum W. H.ex Engl. & $\mathrm{NC}$ & $\mathrm{CL}$ & AG & 117,2 & & & & & 117,2 & 1,20 \\
\hline $\begin{array}{l}\text { Tapirira guianensis Aublet } \\
\text { ANNONACEAE }\end{array}$ & $\mathrm{P}$ & PI & $\mathrm{AG}$ & 44,8 & 3,5 & & & & 48,3 & 1,28 \\
\hline $\begin{array}{l}\text { Guatteria poeppigiana Mart. } \\
\text { ARECACEAE }\end{array}$ & $\mathrm{NC}$ & $\mathrm{CL}$ & $\mathrm{AG}$ & 624,1 & 86,2 & 17,2 & 65,5 & 6,9 & 800,0 & 12,32 \\
\hline Astrocaryum mumbaca Mart. & $\mathrm{NC}$ & $\mathrm{CS}$ & AL & 3,5 & 3,5 & 3,5 & 3,5 & & 13,8 & 0,69 \\
\hline Astrocaryum murumuru Mart. & $\mathrm{NC}$ & $\mathrm{CL}$ & AG & 1762,1 & 1072,4 & 1158,6 & & & 3993,1 & 24,48 \\
\hline Euterpe oleracea Mart. & $\mathrm{C}$ & $\mathrm{CL}$ & $\mathrm{AG}$ & 1113,8 & 686,2 & 103,5 & 244,8 & 193,1 & 2341,4 & 63,31 \\
\hline Geonoma laxiflora Mart. & $\mathrm{NC}$ & $\mathrm{CS}$ & AG & 1803,5 & 944,8 & & & & 2748,3 & 20,10 \\
\hline Jessenia bataua Bur. & $\mathrm{NC}$ & $\mathrm{CS}$ & AG & 158,6 & 37,9 & & 6,9 & 3,5 & 206,9 & 3,44 \\
\hline Oenocarpus bacaba Mart. & $\mathrm{NC}$ & $\mathrm{CL}$ & AG & 51,7 & 17,2 & & 13,8 & 6,9 & 89,7 & 3,78 \\
\hline $\begin{array}{l}\text { Socratea exorrhiza H. Wendl. } \\
\text { BIGNONIACEAE }\end{array}$ & $\mathrm{NC}$ & CS & AG & 55,2 & 31,0 & & & & 86,2 & 1,58 \\
\hline $\begin{array}{l}\text { Jacaranda copaia } \text { D. Don } \\
\text { BURSERACEAE }\end{array}$ & $\mathrm{C}$ & $\mathrm{CL}$ & $\mathrm{AG}$ & 27,6 & & & & & 27,6 & 0,43 \\
\hline Protium cf. krukoffii Swart. & $\mathrm{NC}$ & $\mathrm{CS}$ & - & 3,5 & & & & & 3,5 & 0,14 \\
\hline $\begin{array}{l}\text { Protium spruceanum Engl. } \\
\text { CECROPIACEAE }\end{array}$ & $\mathrm{NC}$ & $\mathrm{CS}$ & $\mathrm{AG}$ & 275,9 & 44,8 & 10,3 & 17,2 & & 348,3 & 5,97 \\
\hline $\begin{array}{l}\text { Cecropia palmata Willd. } \\
\text { CHRYSOBALANACEAE }\end{array}$ & $\mathrm{NC}$ & PI & - & 3,5 & & & & & 3,5 & 0,14 \\
\hline Licania canescens $\mathrm{R}$. Ben. & $\mathrm{NC}$ & $\mathrm{CS}$ & AG & 631,0 & 89,7 & 10,3 & 24,1 & 10,3 & 765,5 & 10,92 \\
\hline Licania cf. octandra O. Kuntze & $\mathrm{NC}$ & $\mathrm{CS}$ & $\mathrm{AG}$ & 13,8 & & & & & 13,8 & 0,33 \\
\hline Licania heteromorpha Benth. & $\mathrm{NC}$ & $\mathrm{CL}$ & $\mathrm{AG}$ & 748,3 & 27,6 & 6,9 & 20,7 & 6,9 & 810,4 & 10,63 \\
\hline Licania macrophylla Benth. & $\mathrm{NC}$ & $\mathrm{CL}$ & $\mathrm{AG}$ & 282,8 & 3,5 & 3,5 & & & 289,7 & 3,49 \\
\hline $\begin{array}{l}\text { Parinari excelsa Sabine } \\
\text { CLUSIACEAE }\end{array}$ & $\mathrm{NC}$ & $\mathrm{CS}$ & $\mathrm{AG}$ & 65,5 & 3,5 & & & & 69,0 & 1,90 \\
\hline Calophyllum brasiliense Camb. & $\mathrm{P}$ & $\mathrm{CS}$ & $\mathrm{AL}$ & 13,8 & 3,5 & 3,5 & & & 20,7 & 0,71 \\
\hline Caraipa grandiflora Mart. & $\mathrm{P}$ & $\mathrm{CS}$ & - & & & & 3,5 & & 3,5 & 0,28 \\
\hline
\end{tabular}

TABELA 1: Continuação ... 
TABLE 1: Continued ...

\begin{tabular}{|c|c|c|c|c|c|c|c|c|c|c|}
\hline \multirow{2}{*}{$\begin{array}{l}\text { Família Botânica e } \\
\text { Nome Científico }\end{array}$} & \multirow{2}{*}{ GU } & \multirow{2}{*}{ GE } & \multirow{2}{*}{ PDE } & \multicolumn{6}{|c|}{ Número de Plantas/ha } & \multirow{2}{*}{ IVIARN } \\
\hline & & & & CT 1 & CT 2 & CT 3 & CT 4 & CT 5 & Total & \\
\hline Rheedia macrophylla $\mathrm{Pl}$. et Tr. & $\mathrm{NC}$ & $\mathrm{CL}$ & $\mathrm{AG}$ & 165,5 & 10,3 & 3,5 & 6,9 & 3,5 & 189,7 & 4,30 \\
\hline Symphonia globulifera L. F. & $\mathrm{P}$ & $\mathrm{CL}$ & $\mathrm{AG}$ & 544,8 & 31,0 & 10,3 & 10,3 & 17,2 & 613,8 & 11,05 \\
\hline $\begin{array}{l}\text { Vismia macrophylla Kunth } \\
\text { COMBRETACEAE }\end{array}$ & $\mathrm{NC}$ & $\mathrm{P}$ & $\mathrm{AL}$ & 10,3 & & & & & 10,3 & 0,43 \\
\hline Cacoucia coccinea Aubl. & $\mathrm{NC}$ & $\mathrm{CS}$ & $\mathrm{AG}$ & 65,5 & 17,2 & 17,2 & 17,2 & & 117,2 & 3,22 \\
\hline $\begin{array}{l}\text { Terminalia dichotoma G. F. W. May. } \\
\text { EUPHORBIACEAE }\end{array}$ & $\mathrm{P}$ & $\mathrm{CL}$ & $\mathrm{AG}$ & 31,0 & & & & & 31,0 & 0,69 \\
\hline Hevea brasiliensis M. Arg. & $\mathrm{NC}$ & $\mathrm{CS}$ & $\mathrm{AG}$ & 27,6 & 6,9 & 3,5 & 6,9 & & 44,8 & 1,39 \\
\hline Hieronyma laxiflora Müll. Arg. & $\mathrm{P}$ & $\mathrm{CL}$ & $\mathrm{AG}$ & 55,2 & & & & & 55,2 & 0,75 \\
\hline $\begin{array}{l}\text { Manihot brachyloba Müll. Arg. } \\
\text { HUMIRIACEAE }\end{array}$ & $\mathrm{NC}$ & $\mathrm{P}$ & $\mathrm{AG}$ & 100,0 & 31,0 & 10,3 & 31,0 & & 172,4 & 3,96 \\
\hline $\begin{array}{l}\text { Sacoglottis guianensis Bentham } \\
\text { ICACINACEAE }\end{array}$ & $\mathrm{P}$ & $\mathrm{CS}$ & $\mathrm{AL}$ & 31,0 & 20,7 & 3,5 & & & 55,2 & 1,61 \\
\hline $\begin{array}{l}\text { Poraqueiba paraensis Ducke } \\
\text { LAURACEAE }\end{array}$ & $\mathrm{NC}$ & $\mathrm{CL}$ & $\mathrm{AL}$ & 10,3 & 6,9 & & & & 17,2 & 0,69 \\
\hline $\begin{array}{l}\text { Nectandra } \text { cf. risi Miq. } \\
\text { LECYTHIDACEAE }\end{array}$ & $\mathrm{NC}$ & $\mathrm{CL}$ & $\mathrm{AG}$ & 210,3 & 82,8 & 20,7 & 24,1 & & 337,9 & 6,01 \\
\hline Eschweilera coriacea Mart. ex Berg. & $\mathrm{NC}$ & $\mathrm{CL}$ & $\mathrm{AG}$ & 127,6 & 51,7 & 31,0 & 34,5 & 13,8 & 258,6 & 7,84 \\
\hline $\begin{array}{l}\text { Gustavia augusta L. } \\
\text { LEGUMINOSAE }\end{array}$ & $\mathrm{NC}$ & CS & AG & 320,7 & 41,4 & 10,3 & 13,8 & 6,9 & 393,1 & 7,58 \\
\hline Crudia bracteata Benth. & $\mathrm{NC}$ & $\mathrm{CS}$ & $\mathrm{AG}$ & 500,0 & 82,8 & 13,8 & 34,5 & 3,5 & 634,5 & 9,79 \\
\hline Dialium guianensis Steud. & $\mathrm{NC}$ & $\mathrm{CS}$ & $\mathrm{AG}$ & 6,9 & 6,9 & 3,5 & 6,9 & & 24,2 & 0,92 \\
\hline Hymenaea oblongifolia Huber & $\mathrm{C}$ & CL & $\mathrm{AG}$ & 34,5 & & 3,5 & & & 37,9 & 0,97 \\
\hline Inga alba Willd. & $\mathrm{NC}$ & $\mathrm{CL}$ & $\mathrm{AG}$ & 82,8 & 27,6 & 3,5 & 3,5 & 6,9 & 124,2 & 4,60 \\
\hline Inga edulis Mart. & $\mathrm{NC}$ & $\mathrm{CL}$ & $\mathrm{AG}$ & 562,1 & 44,8 & 17,2 & 13,8 & 6,9 & 644,8 & 9,35 \\
\hline Inga velutina Willd. & $\mathrm{NC}$ & $\mathrm{CL}$ & $\mathrm{AG}$ & 17,2 & & & 6,9 & & 24,1 & 1,18 \\
\hline Macrolobium augustifolium Cowan & $\mathrm{P}$ & $\mathrm{CS}$ & - & & 3,5 & & & & 3,5 & 0,13 \\
\hline Ormosia coccinea Jack. & $\mathrm{NC}$ & $\mathrm{CL}$ & $\mathrm{AL}$ & & 3,5 & 3,5 & & & 6,9 & 0,26 \\
\hline Ormosia coutinhoi Ducke & $\mathrm{P}$ & CL & $\mathrm{AL}$ & 13,8 & & & & 3,5 & 17,2 & 1,02 \\
\hline Pentaclethra macroloba O. Kuntze & $\mathrm{P}$ & $\mathrm{CS}$ & $\mathrm{AG}$ & 62,1 & & & 6,9 & & 69,0 & 1,81 \\
\hline Pithecellobium juruanum Harms. & $\mathrm{NC}$ & CL & $\mathrm{AL}$ & 13,8 & & & 3,5 & & 17,2 & 0,73 \\
\hline Platymiscium trinitatis Benth. & $\mathrm{P}$ & $\mathrm{CL}$ & $\mathrm{AG}$ & 17,2 & & 3,5 & & & 20,7 & 0,61 \\
\hline Swartzia acuminata Willd. ex Vog. & $\mathrm{NC}$ & CL & $\mathrm{AL}$ & 6,9 & & & & & 6,9 & 0,28 \\
\hline Swartzia racemosa Benth. & $\mathrm{NC}$ & $\mathrm{CS}$ & $\mathrm{AG}$ & 169,0 & 6,9 & 6,9 & & 3,5 & 186,2 & 4,28 \\
\hline Tachigali myrmecophyla Ducke & $\mathrm{NC}$ & $\mathrm{CS}$ & $\mathrm{AG}$ & 203,5 & 10,3 & 6,9 & 27,6 & 13,8 & 262,1 & 7,83 \\
\hline $\begin{array}{l}\text { Vatairea guianensis Aubl. } \\
\text { MELASTOMATACEAE }\end{array}$ & $\mathrm{NC}$ & $\mathrm{P}$ & $\mathrm{AL}$ & 3,5 & 3,5 & & & & 6,9 & 0,27 \\
\hline Miconia ceramicarpa Cogn. & $\mathrm{NC}$ & $\mathrm{CS}$ & $\mathrm{AG}$ & 679,3 & 317,2 & 13,8 & & & 1010,3 & 9,52 \\
\hline $\begin{array}{l}\text { Mouriri grandiflora } \text { DC. } \\
\text { MELIACEAE }\end{array}$ & $\mathrm{NC}$ & $\mathrm{CS}$ & $\mathrm{AG}$ & 31,0 & 20,7 & 3,5 & 3,5 & & 58,6 & 1,22 \\
\hline Carapa guianensis Aublet & $\mathrm{C}$ & $\mathrm{CL}$ & $\mathrm{AG}$ & 58,6 & & 3,5 & & 3,5 & 65,5 & 2,24 \\
\hline Cedre & $\mathrm{C}$ & $\mathrm{CL}$ & $\mathrm{AL}$ & 3,5 & 3,5 & & & & 6,9 & 0,27 \\
\hline $\begin{array}{l}\text { Guarea guidonia Sleumer } \\
\text { MYRISTICACEAE }\end{array}$ & $\mathrm{NC}$ & $\mathrm{CS}$ & $\mathrm{AG}$ & 310,3 & 48,3 & 13,8 & 69,0 & 20,7 & 462,1 & 12,61 \\
\hline Iryanthera sagotiana Warb. & $\mathrm{NC}$ & $\mathrm{C}$ & $\mathrm{AL}$ & 10,3 & & & 3,5 & & 13,8 & 0,71 \\
\hline Virola sp. & $\mathrm{C}$ & CL & - & 3,5 & & & & & 3,5 & 0,14 \\
\hline Virola surinamensis Warb. & $\mathrm{C}$ & $\mathrm{CL}$ & AG & 10303,5 & 27,6 & 10,3 & 3,5 & & 10348,3 & 79,18 \\
\hline
\end{tabular}

TABELA 1: Continuação ... 
TABLE 1: Continued ...

\begin{tabular}{|c|c|c|c|c|c|c|c|c|c|c|}
\hline \multirow{2}{*}{$\begin{array}{l}\text { Família Botânica e } \\
\text { Nome Científico }\end{array}$} & \multirow{2}{*}{ GU } & \multirow{2}{*}{ GE } & \multirow{2}{*}{ PDE } & \multicolumn{6}{|c|}{ Número de Plantas/ha } & \multirow{2}{*}{ IVIARN } \\
\hline & & & & CT 1 & CT 2 & CT 3 & CT 4 & CT 5 & Total & \\
\hline \multicolumn{11}{|l|}{ MYRTACEAE } \\
\hline Eugenia brownsbergii Amshoff & $\mathrm{NC}$ & $\mathrm{CL}$ & $\mathrm{AG}$ & 431,0 & 179,3 & 24,1 & 20,7 & & 655,2 & 8,29 \\
\hline $\begin{array}{l}\text { Eugenia sp } \\
\text { OLACACEAE }\end{array}$ & $\mathrm{NC}$ & $\mathrm{CL}$ & $\mathrm{AL}$ & 13,8 & & & & & 13,8 & 0,57 \\
\hline $\begin{array}{l}\text { Minquartia guianensis Aubl. } \\
\text { SAPOTACEAE }\end{array}$ & $\mathrm{NC}$ & $\mathrm{CS}$ & $\mathrm{AL}$ & 13,8 & 3,5 & & & & 17,2 & 0,58 \\
\hline $\begin{array}{l}\text { Sarcaulus brasiliensis Eyma. } \\
\text { Simaroubaceae }\end{array}$ & $\mathrm{NC}$ & $\mathrm{CS}$ & $\mathrm{AG}$ & 313,8 & 62,1 & 17,2 & 24,1 & & 417,2 & 6,54 \\
\hline $\begin{array}{l}\text { Simaruoba amara Aublet } \\
\text { STERCULIACEAE }\end{array}$ & $\mathrm{C}$ & PI & $\mathrm{AG}$ & 55,2 & & & & & 55,2 & 1,34 \\
\hline Herrania mariae Schum. & $\mathrm{NC}$ & PI & $\mathrm{AL}$ & 65,5 & 20,7 & 3,5 & 10,3 & & 100,0 & 3,21 \\
\hline Sterculia pruriens K. Sch. & $\mathrm{P}$ & $\mathrm{CL}$ & $\mathrm{AL}$ & 3,5 & & & 3,5 & & 6,9 & 0,38 \\
\hline $\begin{array}{l}\text { Theobroma subincanum Mart. } \\
\text { TILIACEAE }\end{array}$ & $\mathrm{NC}$ & $\mathrm{CS}$ & $\mathrm{AG}$ & 327,6 & 24,1 & 3,5 & 17,2 & 13,8 & 386,2 & 8,75 \\
\hline Apeiba burchelii Sprague & $\mathrm{NC}$ & $\mathrm{CL}$ & $\mathrm{AG}$ & 20,7 & 3,5 & 3,5 & & & 27,6 & 0,65 \\
\hline Indeterminada 1 & $\mathrm{NC}$ & $\mathrm{CS}$ & $\mathrm{AG}$ & 3,5 & 17,2 & 3,5 & 3,5 & & 27,6 & 0,50 \\
\hline Indeterminada 2 & $\mathrm{NC}$ & $\mathrm{CL}$ & - & 3,5 & & & & & 3,5 & 0,14 \\
\hline Total & - & - & - & 23931,0 & 4289,7 & 1589,7 & 810,4 & 348,33 & 30969,0 & 400,00 \\
\hline
\end{tabular}

Com relação às espécies não-comerciais, verificou-se que seis são espécies pioneiras, vinte clímax exigentes de luz e 24 clímax tolerantes à sombra. Por conseguinte, das 20 espécies comerciais e potenciais que poderão fazer parte do estoque de crescimento $(15 \mathrm{~cm} \leq \mathrm{DAP}<45 \mathrm{~cm}$ ) e exploração (DAP $\geq 45 \mathrm{~cm}$ ), $65 \%$ são clímax exigente de luz e $10 \%$ são pioneiras o que é uma característica notadamente desejável, quando se executa um plano de manejo por meio do corte seletivo, porque esse sistema de manejo abre pequenas clareiras na floresta e, conseqüêntemente, aumenta a disponibilidade de luz no sub-bosque, favorecendo o crescimento e estabelecimento da regeneração das espécies pioneiras e clímax exigentes de luz.

A análise de regressão com resposta em platô confirmou que a amostra utilizada (29 parcelas) foi suficiente para representar a composição florística, indicando que o lançamento de apenas 13 parcelas (0,13 ha) já seria o suficiente para amostrar a composição florística da regeneração natural (Figura 1).

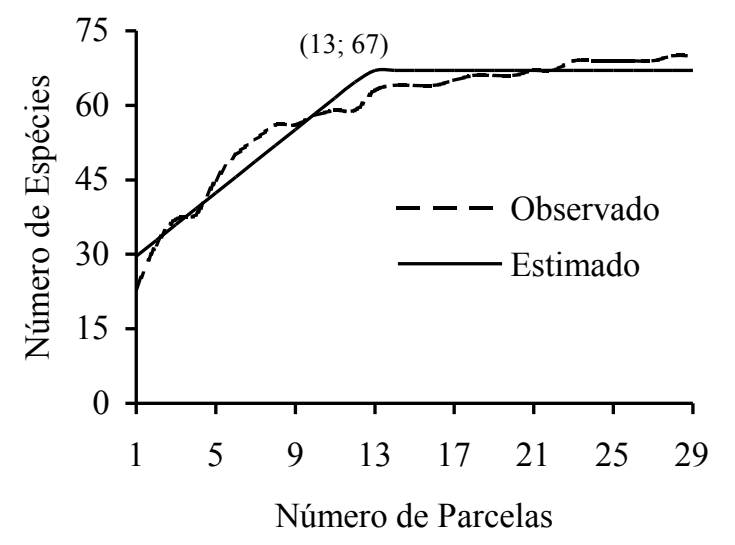

FIGURA 1: Suficiência amostral para regeneração natural de várzea alta inventariada na propriedade florestal da EMAPA, município de Afuá, Pará.

FIGURE 1: Sample sufficiency for the natural regeneration of an upland floodplain forest inventoried in EMAPA forestlands, Afuá County, State of Pará. 
O índice de diversidade de Shannon (H') para a vegetação estudada foi 2,68. Bentes-Gama (2000), analisando o estrato arbóreo (DAP $\geq 15 \mathrm{~cm}$ ) na mesma área de estudo, encontrou maior diversidade $\left(\mathrm{H}^{\prime}=3,62\right)$, enquanto Macedo (1996) obteve um $\mathrm{H}^{\prime}=2,76$ para floresta de várzea não-explorada no estuário amazônico. Bentes e Maciel (1994), inventariando a vegetação com DAP $\geq 10 \mathrm{~cm}$ de floresta de várzea explorada no município de Afuá, encontraram no rio Cajuúna, $\mathrm{H}^{\prime}=2,56$, e no rio Urucu, $\mathrm{H}^{\prime}=3,17$. Foi possível verificar, assim, que os valores de diversidade são influenciados pelo nível de inclusão do inventário e pelo histórico de uso da floresta.

Kalliola et al. (1993) citam que a floresta de várzea apresenta menor diversidade que a floresta de terra firme, uma vez que abriga animais e plantas adaptados a condições hidrológicas sazonais. Silva et al. (1992) comentam que essa baixa diversidade ocorre, porque poucas espécies dispõem de mecanismos morfofisiológicos que tolerem o ritmo sazonal de inundação.

Ao se comparar a composição florística da regeneração natural com a do estrato arbóreo (DAP $\geq 15 \mathrm{~m}$ ) inventariado por Bentes-Gama (2000), verificou-se que 75\% das espécies identificadas na área de estudo ocorreram nos dois estratos.

Entre as espécies arbóreas que só ocorreram na regeneração, destacaram-se: Caraipa grandiflora, Macrolobium augustifolium, Pithecellobium juruanum e Vatairea guianensis. Espera-se que com o decorrer do desenvolvimento natural da floresta ou mediante ações de manejo, tais espécies ingressem nos estratos médio e superior da floresta.

Como espécies de pequeno porte, por não alcançarem diâmetros superiores a $15 \mathrm{~cm}$, e que foram consideradas como regeneração natural, destacaram-se: Astrocaryum mumbaca, Cacoucia coccinea, Dialium guianensis, Geonoma laxiflora, Herrania mariae e Trichanthera gigantea.

Verificou-se que algumas espécies não tiveram representação nas classes de tamanho de regeneração natural estudadas, como foi o caso de: Allantoma lineata, Brosimum rubescens, Campsiandra laurifolia, Ceiba pentandra, Couratari guianensis, Diplotropis martiusii, Eugenia floribunda, Ficus gomelleira, Gomphia castaneaefolia, Lecythis lurida, Mora paraensis, Pseudima frutescens, Pterocarpus amazonicus, Sapium cf. hippomane, Schefflera paraensis, Taralea oppositifolia e Vochysia guianensis. Essas espécies apresentaram indivíduos apenas no estrato arbóreo, conforme o levantamento florístico realizado por Bentes-Gama (2000). Esse resultado só vem a reforçar a necessidade de se realizar a condução da regeneração natural dessas espécies, pois, uma vez que estas não encontrem condições ambientais adequadas para germinarem e se estabelecerem, poderão ser fatalmente extintas da área.

\section{Análise estrutural}

A densidade total da regeneração natural foi 30.969 indivíduos/ha, com área basal de $7,18 \mathrm{~m}^{2} /$ ha para o intervalo de $\mathrm{h} \geq 3,0 \mathrm{~m}$ até DAP $<15,0 \mathrm{~cm}$. De acordo com Rodrigues (1961), a alta densidade de plantas na regeneração natural de várzea alta está diretamente correlacionada com as condições edafoclimáticas e a sazonalidade das inundações. A altura média foi de 1,2 m e máxima de $15 \mathrm{~m}$. As espécies, que apresentaram as maiores alturas $(\mathrm{h} \geq 12 \mathrm{~m})$, foram: Euterpe oleracea, Tachigalia myrmecophila, Inga edulis e Eschweilera coriacea. Como se pode verificar na Tabela 1, todas essas espécies inventariadas são clímax tolerantes à sombra, com exceção de Euterpe oleracea, o que indica que elas se desenvolvem bem em condições naturais de sub-bosque (pouca luminosidade). Albuquerque (1999) comenta que as espécies clímax tolerantes à sombra são muito sensíveis à exploração florestal de alto impacto, por ela provocar grandes clareiras e com isso aumentar significativamente a disponibilidade de luz no interior da floresta. Sendo este, portanto, um dos motivos para não se utilizar tal forma de exploração em florestas tropicais como as várzeas.

A distribuição do número de indivíduos por classe de tamanho (Figura 2) demonstrou a tendência esperada de diminuição do número de plantas e espécies com o aumento das classes, esses resultados são compatíveis com os encontrados por Vieira e Hosokawa (1989) para florestas de terra firme não-exploradas, com $25 \%$ e $75 \%$ de redução de área basal após um ano de exploração. 


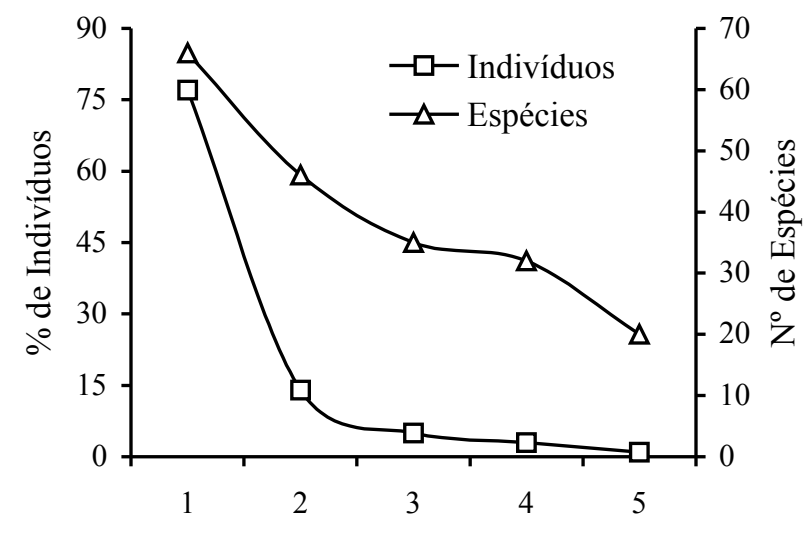

Classe de Tamanho

FIGURA 2: Número de espécies e percentual de indivíduos por classe de tamanho inventariada na propriedade florestal da EMAPA, município de Afuá, Pará.

FIGURE 2: Number of species and percentual distribution of individuals by class size inventoried in EMAPA forestlands, Afuá County, State of Pará.

As espécies, que mais se destacaram na comunidade estudada, foram: Virola surinamensis, Euterpe oleracea, Astrocaryum murumuru, Geonoma laxiflora, Guarea guidonia, Guatteria poeppigiana, Symphonia globulifera, Licania canescens, Licania heteromorpha, Crudia bracteata, Miconia ceramicarpa, Inga edulis, Theobroma subicanum e Eugenia brownsbergii (Tabela 1). Essas espécies foram responsáveis por $72,6 \%$ do índice de valor de importância ampliado; como em outras florestas da Amazônia poucas espécies dominam a vegetação.

As duas espécies mais importantes, Virola surinamensis e Euterpe oleracea, são as mais comercializadas no estuário amazônico. Virola surinamensis tem a sua madeira aproveitada por serrarias e indústrias de compensado, e de Euterpe oleracea comercializa-se o fruto e o palmito de açaí, que é o segundo produto tradicional mais explorado nas áreas de várzea do estuário amazônico. Entre as espécies potenciais, apenas Symphonia globulifera teve destaque cuja madeira é utilizada em construção de casas pelos ribeirinhos.

Em relação às espécies não-comerciais, que desempenham importantes funções ambientais e socioeconômicas na área de estudo, estão: Astrocaryum murumuru, Miconia ceramicarpa, Inga edulis e Guarea guidonia cujos frutos são consumidos pela fauna; Geonoma laxiflora que tem suas folhas utilizadas em cobertura de casas; Guarea guidonia cuja madeira é utilizada em construções rústicas e de canoas. Ainda para construções rústicas, verificou-se o uso das madeiras de Guatteria poeppigiana, Licania canescens, Licania heteromorpha e Miconia ceramicarpa, e Crudia bracteata cujos indivíduos de pequeno porte são muito utilizados como varas para empurrar pequenas embarcações.

Mediante um plano de manejo florestal, uma das espécies que poderiam ser exploradas comercialmente para a produção de madeira, por apresentar regeneração natural suficiente para garantir a reposição do estoque de exploração (DAP $\geq 45 \mathrm{~cm}$ ), é Virola surinamensis que apresentou 10.348 indivíduos/ha e ocorreu em todas as unidades de amostra. Entretanto, verificou-se que 99,57\% de seus indivíduos estavam concentrados na CT 1 o que fez com que esta apresentasse baixa dominância $(0,85 \%)$. Observou-se no local que a maior parte de suas mudas não estavam recebendo intensidade de luz adequada para ingressarem nas classes de tamanho subseqüentes, pelo fato de estarem concentradas sob a sombra de árvores matrizes. Symphonia globulifera foi outra espécie que se destacou na regeneração natural apresentando 613,79 indivíduos/ha distribuídos satisfatoriamente entre as classes de tamanho os quais ocorreram em 96,5\% das unidades amostrais.

Outra espécie de uso múltiplo, grande retorno econômico, e viável para ser incluída em planos de manejo é Euterpe oleracea que se destaca no mercado nacional e internacional pela produção de palmito e 
fruto de açaí. Essa espécie apresentou 2.341 indivíduos/ha, ocorreu em todas as unidades de amostra, teve uma boa distribuição dos indivíduos entre as classes de tamanho (47,57\% na CT 1, 29,31\% na CT 2, 4,42\% na CT 3, 10,46\% na CT 4 e 8,25\% na CT5) o que fez com que esta apresentasse a maior dominância e o segundo maior índice de valor de importância ampliado (Tabela 1).

Quando da aplicação de tratamentos silviculturais na floresta, a regeneração natural de Virola surinamensis, Symphonia globulifera e Euterpe oleracea pode ser facilmente conduzida para a formação de bons estoque de crescimento e exploração, considerando que apresentam grande potencialidade para a propagação sexuada. Uma sugestão seria diminuir o número de indivíduos (refinamento) na CT1, e realizar a abertura de dossel (liberação) para induzir o crescimento das mudas e varas dessas espécies. Assim, espera-se aumentar a movimentação dos indivíduos para as sucessivas classes de tamanho.

Entre as espécies não-comerciais que apresentaram densidade satisfatória (DA $\geq 90$ indivíduos/ha) e ocorreram na maioria das classes de tamanho, são consideradas importantes para a manutenção da fauna silvestre, como também são aproveitadas pelos ribeirinhos, pode-se destacar: Tachigalia mymercophila e Gustavia augusta - madeira utilizada em construções rústicas; Sarcaulus brasiliensis, Theobroma subicanum, Herrania mariae, Oenocarpus bacaba, Inga alba, Jessenia bataua e Rheedia macrophylla frutos são consumidos pelo homem e por animais silvestres; Nectandra cf. risi - madeira utilizada na construção de barcos e móveis; Eschweilera coriacea - entre casca utilizada na fabricação de cordas; Cacoucia coccínea - madeira utilizada para confeccionar cabo de machado; Manihot brachyloba - madeira para lenha e ainda é plantada para a formação de cercas vivas; e Protium spruceanum - resina utilizada para calafetar embarcações.

De modo geral, entre as espécies que foram citadas, poucas apresentam condições de serem aproveitadas em projetos que visem apenas a produção de madeira. Entretanto, verificou-se que há inúmeras espécies que podem ser utilizadas seja para à obtenção de produtos não-madeireiros, ou ainda substituindo a madeira de espécies que já têm um mercado definido por aquelas que já vêm sendo utilizadas para o mesmo fim. Há, portanto, necessidade de efetuar estudos que confirmem suas propriedades tecnológicas e divulguem amplamente suas características dendrológicas.

Com base no trabalho de Martini et al. (1998), as espécies potencialmente suscetíveis aos impactos da exploração foram Minquartia guianensis e Calophyllum brasiliense. Pode-se ainda incluir, nesta lista, algumas espécies que apresentaram densidade muito baixa (DA $<10$ indivíduos/ha) na regeneração natural e foram consideradas raras (DA $<1$ indivíduo/ha) por Bentes-Gama (2000) no estrato arbóreo, a saber: Caraipa grandiflora, Cedrela odorata, Macrolobium augustifolium e Sterculia pruriens. Como todas essas espécies apresentam madeira de boa qualidade e/ou são atrativas para a fauna em razão das características de seus frutos, deve-se ter especial atenção para elas no momento da exploração, para evitar que sejam extintas da área de estudo.

Quanto à tendência de distribuição espacial das espécies (Tabela 1), verificou-se que 68,5\% apresentaram padrão agregado e $22,9 \%$ aleatório. Não foi calculado o padrão de distribuição espacial para $8,6 \%$ das espécies porque elas só ocorreram em uma parcela. Conforme Nascimento et al. (2001), espécies que apresentam esse tipo de padrão de distribuição necessitam de maior área de amostragem e uma distribuição diferenciada das unidades amostrais para a definição de seu padrão de distribuição espacial. As espécies mais abundantes da comunidade vegetal apresentaram distribuição espacial agregada, assim como em Vieira e Hosokawa (1989) e Santana et al. (1997).

\section{Constituição da regeneração natural}

Fazendo uma análise das formas de vida relacionadas na Figura 3, as árvores e palmeiras representaram $36,1 \%$ da densidade relativa, os arbustos $21,4 \%$, as herbáceas $18,6 \%$ e os cipós $23,9 \%$. Esses resultados diferem dos apresentados por Macedo (1996) que estudou a regeneração natural de floresta de várzea no estuário amazônico e verificou que as árvores e palmeiras representaram $49 \%$ da densidade relativa, os arbustos $1 \%$, as herbáceas (ervas e gramíneas) $42 \%$ e os cipós $8 \%$. No trabalho de Rodrigues (1961), realizado em uma floresta de várzea alta no baixo rio Negro, estado do Amazonas, a regeneração natural $(0,1 \mathrm{~m} \leq \mathrm{h} \leq 10,0 \mathrm{~m})$ apresentou $79,4 \%$ de árvores, arbustos e palmeiras; $13,1 \%$ de herbáceas e 7,5\%

Ciência Florestal, v. 13, n. 2, 2003 
de cipós.

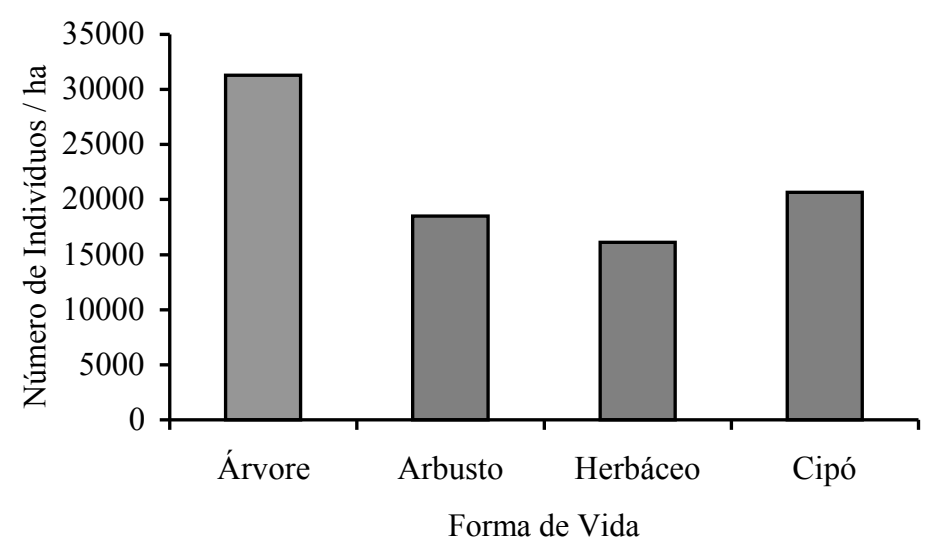

FIGURA 3: Densidade dos indivíduos, por forma de vida, registrados na regeneração natural inventariada na propriedade florestal da EMAPA, município de Afuá, Pará.

FIGURE 3: Distribution of the natural regeneration by life form in the upland floodplain forest inventory in EMAPA forestlands, Afuá County, State of Pará.

Ao se comparar as densidades percentuais referentes às plantas herbáceas e aos cipós deste trabalho com os citados anteriormente, e verificou uma diferença de resultados em razão, provavelmente, do período de coleta de dados. Pois, durante a inundação da várzea, grande parte dos cipós regenerantes morre. Depois que as águas descem, há uma grande proliferação de plantas herbáceas, e antes das inundações as populações de herbáceas e cipós estão bem desenvolvidas, como foi a situação encontrada nesse inventário. Contudo, os resultados foram similares com relação à presença das espécies arbóreas na regeneração natural, ou seja, as árvores e palmeiras são mais abundantes do que as demais formas de vida analisadas. Segundo Meira-Neto e Martins (2000), essa dominância ocorre em razão da grande influência biótica exercida pelos indivíduos arbóreos, provavelmente por meio da chuva de suas sementes.

Segundo Schettino (1999), a alta ocorrência de cipós caracteriza uma forte competição com os indivíduos arbóreos, dificultando o estabelecimento e desenvolvimento das espécies arbóreas regenerantes, sendo essa uma das razões pelas quais os cipós devam ser controlados. O mesmo autor ainda cita que o corte de cipós desencadeia variações positivas nas taxas de regeneração natural, pois promove o aumento do número de indivíduos e da área basal e, conseqüêntemente, eleva a taxa de ingresso de novos indivíduos no estrato arbóreo. Com referência à presença de cipós nos indivíduos com DAP $\geq 15 \mathrm{~cm}$, Bentes-Gama (2000) verificou uma incidência de $31 \%$ o que já era esperado por causa da alta densidade de cipós na regeneração natural. No caso da execução de um plano de manejo florestal, esses dados reforçam a necessidade de aplicação do tratamento silvicultural de cortes de cipós antes da derrubada das árvores.

A qualidade estrutural das plantas arbóreas e das palmeiras foi considerada muito boa, pois mais de $98 \%$ dos indivíduos estavam bem formados e eretos. O percentual de indivíduos caídos (tombados) foi menor que $0,4 \%$ (Tabela 2 ).

Quanto aos indivíduos que apresentaram algum dano estrutural em razão do ataque por animais, (quebrado, roído, cortado, tombado, entre outros) pragas ou doenças, a maior incidência ocorreu entre as espécies não comerciais $(1,57 \%)$, por esse grupo apresentar um maior número de espécies, que são utilizadas como alimento pelos animais silvestres. As espécies não-comerciais que se destacaram pela preferência dos animais, foram Sarcaulus brasiliensis, Eschweilera coriacea e Miconia ceramicarpa. Essas são espécies importantes para a manutenção da fauna local, havendo, porém, a necessidade de estudos mais específicos para a confirmação dessa hipótese.

TABELA 2: Qualidade da regeneração natural das espécies inventariadas na propriedade florestal da EMAPA, município de Afuá, Pará. 
TABLE 2: Quality of the natural regeneration species inventoried in EMAPA forestlands, Afuá County, State of Pará.

\begin{tabular}{l|ccc}
\hline \multirow{2}{*}{ Grupo de Uso } & \multicolumn{3}{|c}{ Qualidade da Regeneração Natural (\%) } \\
\cline { 2 - 4 } & Viva em Pé & Viva Caída & Com Danos \\
\hline Comerciais & 99,52 & 0,13 & 0,35 \\
Potenciais & 98,54 & 0,36 & 1,09 \\
Não comerciais & 98,21 & 0,22 & 1,57 \\
\hline Todas as espécies & 98,76 & 0,19 & 1,05 \\
\hline
\end{tabular}

\section{CONCLUSÕES}

A análise dos resultados deste estudo permitiu concluir que:

Virola surinamensis, Euterpe oleracea, Astrocaryum murumuru, Geonoma laxiflora e Guarea guidonia foram as espécies mais importantes da regeneração natural.

Os cipós estão competindo com os indivíduos arbóreos, sendo, portanto, indicada a realização de cortes de cipós antes de futuras explorações na área.

Deve-se implementar tratamentos silviculturais que beneficiem especialmente o desenvolvimento da regeneração natural de Virola surinamensis e Euterpe oleracea, pois são as espécies comerciais mais valorizadas na região e que apresentaram alta densidade nas menores classes de tamanho.

Muitas das espécies não-comerciais, que estavam bem distribuídas nas classes de tamanho, são importantes para a alimentação da fauna, como também fornecem madeira e produtos florestais não-madeireiros para a subsistência dos ribeirinhos. O aproveitamento desdas espécies poderá ampliar as alternativas de uso dos recursos florestais e agregar valor à floresta por meio do manejo e comercialização de produtos florestais madeireiros e não-madeireiros.

\section{REFERÊNCIAS BIBLIOGRÁFICAS}

ALBUQUeRQUE, G. B. de. Floresta Nacional de Ipanema: caracterização da vegetação em dois trechos distintos do Morro de Araçoiaba, Iperó (SP). 1999. 186p. Dissertação (Mestrado em Ciências Florestais) - Escola Superior de Agricultura Luiz de Queiroz, Piracicaba, 1999.

BENTES, M. P. de M., MACIEL, U. N. Composição florística, estrutura e multipropósito de espécies arbóreas de mata de várzea no município de Afuá, arquipélago de Marajó, PA Belém: MPEG-DBO, 1994. 30p.

BENTES-GAMA, M. de M. Estrutura, valoração e opções de manejo sustentado para uma floresta de várzea na Amazônia. 2000. 206p. Dissertação (Mestrado em Engenharia Florestal) - Universidade Federal de Lavras, Lavras, 2000.

CARVALHO, J. O. P. de. Análise estrutural da regeneração natural em floresta tropical densa na região do Tapajós no Estado do Pará. 1982. 128p. Dissertação (Mestrado em Engenharia Florestal) - Universidade Federal do Paraná, Curitiba, 1982.

CRONQUIST, A. The evolution and classification of flowering plants. New York: The New York Botanical Garden, 1988. 555p.

CURTIS, J. T.; McINTOSH, R. P. An upland forest continuum in the prairie forest border region of Wisconsin. Ecology, Oxford, v. 32, p. 476-496, 1951.

FAO. Silvicultural research in the Amazon. Roma: Food and Agriculture Organization of the United Nations, 1971. 192p. (Technical Report, 3).

FINOL, U. H. Nuevos parametros a considerarse en el analisis estructural de las selvas virgines tropicales.Revista Forestal Venezolana, Merida, v. 14, n. 21, p. 29-42, 1971.

IBAMA - INSTITUTO BRASILEIRO DO MEIO AMBIENTE E DOS RECURSOS NATURAIS RENOVÁVEIS. Instrução Normativa $n^{\circ} 4$ de 04/03/02. Brasília: IBAMA, 2002, $31 p$.

KALliOLA, R.; PUHAKKA, M.; DANJOY, W. Amazônia Peruana: vegetación húmeda tropical en el llano sudandino. Finlândia: Gummerus Printing, 1993. 265p.

LUDWIG, J. A.; REYNOLDS, J. F. Statistical ecology: a primer on methods and computing. New York: John Wiley e

Ciência Florestal, v. 13, n. 2, 2003 
Sons, $1988.337 \mathrm{p}$.

MACEDO, D. S. M. dos S. Estrutura e manejo de uma floresta de várzea do estuário amazônico. 1996. 117p. Dissertação (Mestrado em Ciências Florestais) - Escola Superior de Agricultura Luiz de Queiroz, Piracicaba.

MARTINI, A.; ROSA, N. de A.; UHL, C. Espécies de árvores potencialmente ameaçadas pela atividade madeireira na Amazônia. Belém: IMAZON, 1998. 35p. (Série Amazônia, 11).

MEIRA-NETO, J. A. A.; MARTINS, F. R.. Composição florística do estrato herbáceo-arbustivo de uma floresta estacional semidecidual em Viçosa-MG. Revista Árvore, Viçosa, v. 24, n. 4, p. 407-416, 2000.

NAPPO, M. E. Inventário florístico e estrutural da regeneração natural no sub-bosque de povoamentos homogêneos de Mimosa scabrella Bentham implantados em áreas mineradas, em poços de caldas, Minas Gerais. 1999. 87p. Dissertação (Mestrado em Engenharia Florestal) - Universidade Federal de Lavras, Lavras, 1999.

NASCIMENTO, A. R. T.; LONGHI, S. J.; BRENA, D. A. Estrutura e padrões de distribuição espacial de espécies arbóreas em uma amostra de Floresta Ombrófila Mista em Nova Prata, RS. Ciência Florestal, Santa Maria, v. 11, n. 1, p. 105-119, 2001.

OLIVEIRA, L. C. de Dinâmica de crescimento e regeneração natural de uma floresta secundária no Estado do Pará. 1995. 126p. Dissertação (Mestrado em Ciências Biológicas) - Universidade Federal do Pará, Belém, 1995.

OLIVEIRA-FILHO, A. T. Estudos ecológicos da vegetação como subsídio para programas de revegetação com espécies nativas: uma proposta metodológica. Cerne, Lavras, v. 1, n. 1, p. 64-72, 1994.

PRANCE, G. T. Notes on the vegetation of Amazonia III: the terminology of Amazon forest types subject to inundation. Brittonia, New York, v. 31, p. 26-38, 1979.

RODRIGUES, W. A. Estudo preliminar de mata de várzea alta de uma ilha do baixo rio Negro de solo argiloso e úmido. Manaus: INPA, 1961. 50 p. (Série Botânica, 10).

ROLLET, B. La regeneración natural en bosque denso siempreverde de llanura de la Guayana Venezolana. Guayana Venezolana: Centro de Documentación y Publicaciones del IFLAIC, 1969. (IFLAIC, 124).

SANTANA, J. A. da S.; BARROS, L. P.; JARDIM, F. C. da S. Análise da vegetação de regeneração natural na floresta tropical úmida em Paragominas-PA. Boletim da FCAP, Belém, n. 28, p. 9-35, 1997.

SCHETTINO, S. Efeito do corte de cipós sobre a dinâmica de sucessão, crescimento e produção de uma floresta ombrófila densa secundária, na Reserva Florestal de Linhares-ES. 1999. 118p. Dissertação (Mestrado em Ciência Florestal) - Universidade Federal de Viçosa, Viçosa, 1999.

SEITZ, R. A. A análise do povoamento: o primeiro passo. Floresta, Curitiba, v.18, n.1/2, p.4-11, 1988.

SILVA, S. M.; SILVA, F. C.; VIEIRA, A. O. S.; NAKAJIMA, J. N.; PIMENTA, J. A.; COLLI, S. Composição florística e fitossociológica do componente arbóreo das florestas ciliares da bacia do rio Tibagi, Paraná: 2. Várzea do rio Bitumirim, Município de Ipiranga, PR. In: CONGRESSO NACIONAL SOBRE ESSÊNCIAS NATIVAS, 2., 1992, São Paulo. Anais... São Paulo: Instituto Florestal, 1992. p. 192-198.

SUDAM/PROJETO DE HIDROLOGIA E CLIMATOLOGIA DA AMAZÔNIA. Atlas climatológico da Amazônia brasileira. Belém: SUDAM, 1984. 125p.

VIEIRA, G.; HOSOKAWA, R. T. Composição florística da vegetação da regeneração natural 1 ano após diferentes níveis de exploração de uma floresta tropical úmida. Acta Amazonica, Manaus, v. 19, p. 401-413, 1989. 